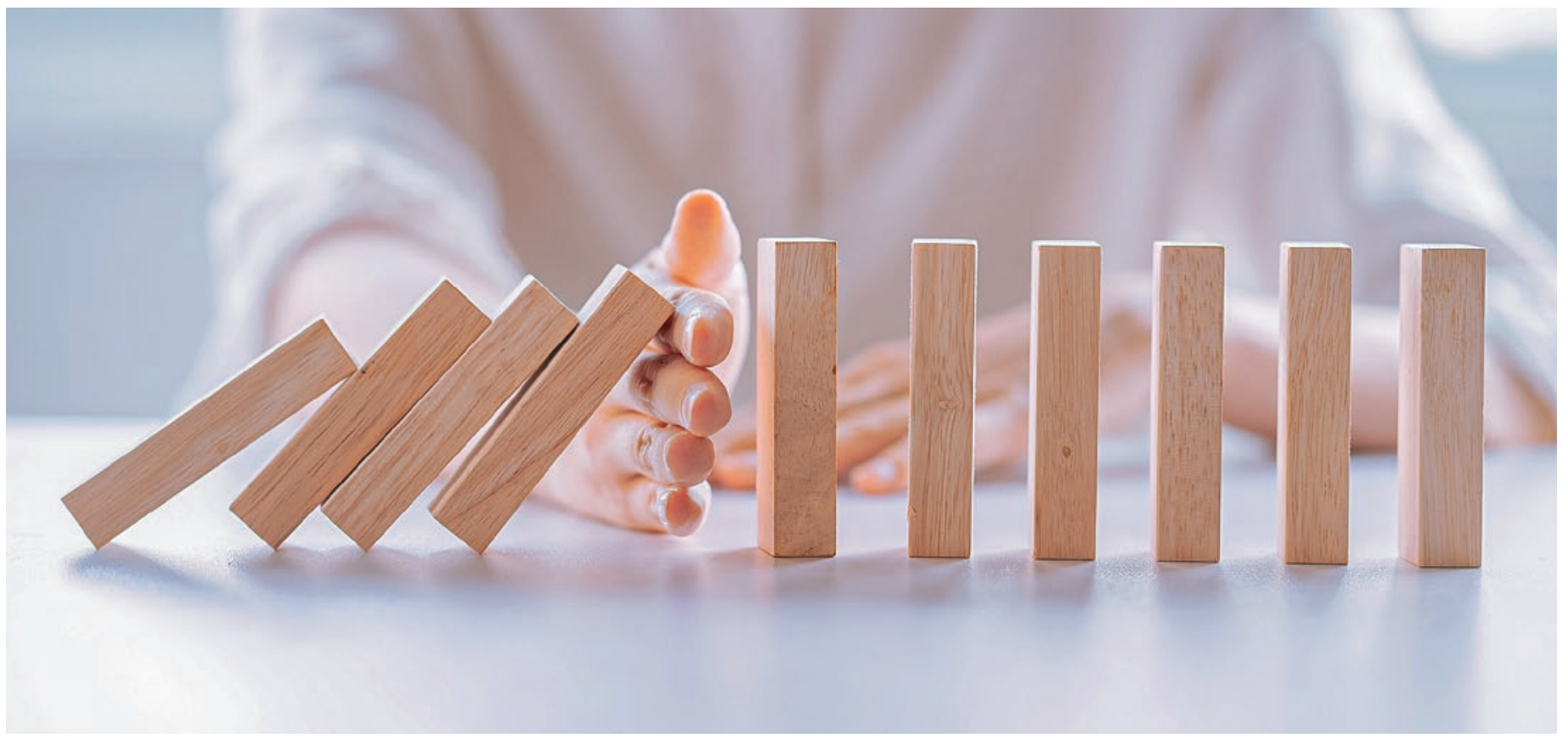

\title{
Wechsel zum Anordnungsmodell: Es gibt noch viel zu tun
}

\section{Fulvia Rota ${ }^{a}$, Rafael Traberb $^{\mathrm{b}}$, Nanda Samimi $^{\mathrm{c}}$, Erich Seifritz ${ }^{\mathrm{d}}$}

${ }^{a}$ Dr. med., Präsidentin der Schweizerischen Gesellschaft für Psychiatrie und Psychotherapie SGPP; b Dr. med., Vizepräsident SGPP; ${ }^{c}$ Eidg. Dipl. pharm. ETH, Vizepräsidentin der Swiss Mental Health Care SMHC; d Prof. Dr. med., Präsident der SMHC

Der Wechsel vom Delegations- zum Anordnungsmodell für die psychologische Psychotherapie erfolgt überstürzt: Die Kantone haben bis jetzt noch immer keine Steuerungsinstrumente in der Hand, und die geplante klinische Weiterbildung ist unzureichend. Diese Punkte müssen geklärt werden, bevor das Anordnungsmodell in Kraft tritt. Sonst droht eine Mengenausweitung, welche die damit angestrebten Versorgungsziele verfehlt.

Am 19. März 2021 gab der Bundesrat bekannt, dass das Delegationsmodell per 1. Juli 2022 durch das Anordnungsmodell ersetzt werden soll. Im neuen Modell können die psychologischen Psychotherapeutinnen und Psychotherapeuten ihre Leistungen selbständig im Rahmen der obligatorischen Krankenpflegeversicherung erbringen.

Als Ziel gab der Bundesrat vor, dass durch den vereinfachten Zugang zur Psychotherapie Versorgungsengpässe in der Kinder- und Jugendpsychiatrie, in Krisenund Notfallsituationen, für schwere psychische Erkrankungen sowie in ländlichen Regionen reduziert werden sollen. Monate später zeigt sich: Das bleibt Wunschdenken.
Die Zeit für die Umsetzung ist viel zu knapp bemessen. Angesichts der Komplexität und Tragweite des Systemwechsels wäre eine Revision des Krankenversicherungsgesetzes (KVG) angezeigt gewesen. Auf dem stattdessen beschrittenen Verordnungsweg sind ein halbes Jahr vor der Umsetzung immer noch essentielle Fragen in Bezug auf die gezielte Versorgung, die Sicherheit, die Qualität und die Kosten offen.

Wegen der vielen Unklarheiten droht nun eine Fehlversorgung mit unkontrolliertem Kostenanstieg. Und dies in einer Zeit, in der die Politik intensiv über die Kostendämpfungspakete I und II diskutiert, die in allen Bereichen des Gesundheitswesen Einsparungen bringen sollen. 


\section{Was politisch gerade geschieht}

Der Bundesrat hat einen Termin für den Systemwechsel festgelegt, obschon die Motion der nationalrätlichen Gesundheitskommission, welche auf Gesetzesebene eine Zulassungssteuerung der Psychologinnen und Psychologen fordert, vom Ständerat noch nicht behandelt wurde. Die Schweizerische Gesellschaft für Psychiatrie und Psychotherapie (SGPP) und die Organisation Swiss Mental Health Care (SMHC) wandten sich deshalb an die Mitglieder der ständerätlichen Gesundheitskommission, worauf am 19. Oktober 2021 eine Anhörung mit den betroffenen Verbänden stattfand. Als Folge davon haben die Kommissionsmitglieder die besagte Motion dem Ständerat zur Annahme empfohlen. Dieser hat am 6. Dezember 2021 die Motion schliesslich an den Bundesrat überwiesen. Anders als der Berufsverband Föderation der Schweizer Psychologinnen und Psychologen (FSP) verlautbaren liess, stand an der Anhörung zur Motion nicht das Anordnungsmodell zur Debatte. Es geht der SGPP und der SMHC ausschliesslich darum, die Voraussetzungen zu schaffen, um mit dem Systemwechsel die Versorgung von Menschen mit psychischen Erkrankungen zu verbessern und keine unzulässigen Mehrkosten zu verursachen.

Da die Motion zur Zulassungssteuerung nicht in dieser kurzen Frist umgesetzt werden kann, hat sich die ständerätliche Gesundheitskommission Mitte November 2021 mit einem Brief an den Bundesrat gewandt. Er wird aufgefordert, bereits im Hinblick auf die Inkraftsetzung der Verordnungsanpassung per 1. Juli 2022 das in seiner Kompetenz Stehende zu tun, um ungerechtfertigten Mengenausweitungen vorzubeugen und eine qualitativ hochstehende Versorgung sicherzustellen.

\section{Es muss noch einiges geklärt werden}

Es braucht jetzt Massnahmen, um nicht im Nachhinein Fehlentwicklungen korrigieren zu müssen. Das sind die offenen Fragen, die zwingend zu klären sind, bevor das Anordnungsmodell in Kraft tritt:

\section{Zulassungssteuerung}

Die Kantone müssen unbedingt in der Lage sein, die Zulassung der psychologischen Psychotherapeutinnen und -therapeuten zu steuern, bevor das Anordnungsmodell in Kraft tritt. In der jetzigen Ausgangslage käme es zu einer flächendeckenden Erhöhung. Das Angebot in den Städten, das bereits ausreichend ist, würde weiter ausgebaut. Das führt unweigerlich zu einer Mengenausweitung, ohne dass dort, wo heute tatsächlich Engpässe bestehen, die Versorgung verbessert wird: in der Kinder- und Jugendpsychiatrie, für Menschen mit schweren psychischen Erkrankungen, in Randregionen und in der Notfall- und Krisenpsychiatrie.

Die bereits bestehenden personellen Engpässe in den psychiatrischen Institutionen mit ihrer umfassenden Versorgungspflicht drohen sich zudem weiter zu verschärfen, wenn der Zugang zum ambulanten Markt unreguliert bleibt.

Jedes Jahr schliessen rund 400 Psychologinnen und Psychologen ihre Weiterbildung ab, die sie zur selbständigen Ausübung der psychologischen Psychotherapie berechtigt. Zum Vergleich: Die Zahl der Ärztinnen und Ärzte, die jedes Jahr ihre Weiterbildung zum

\section{Angesichts der Komplexität und Tragweite des} Systemwechsels wäre eine Revision des Krankenversicherungsgesetzes angezeigt gewesen.

Facharzt bzw. zur Fachärztin für Psychiatrie und Psychotherapie abschliessen und in zweifacher Hinsicht reguliert sind, ist nicht einmal halb so gross.

\section{Klinische Weiterbildung}

Bevor das Anordnungsmodell in Kraft tritt, muss die klinisch-praktische Weiterbildung der psychologischen Psychotherapeutinnen und -therapeuten geregelt sein: Bis heute sind weder die Inhalte definiert, noch ist die Finanzierung gesichert. Die Krankenpflege-Leistungsverordnung (KLV) und die Krankenversicherungsverordnung sehen zudem lediglich ein Jahr klinisch-praktische Erfahrung in einer vom Schweizerischen Institut für ärtzliche Weiter- und Fortbildung (SIWF) zertifizierten Weiterbildungsstätte vor. Das ist für die angestrebte, weitgehend selbständige Behandlung von psychischen Erkrankungen ungenügend.

Selbständig tätige Psychologinnen und Psychologen müssen in ihrer Weiterbildung das gesamte Spektrum psychischer Erkrankungen kennenlernen. Denn im neuen Anordnungsmodell kommt die Psychiaterin bzw. der Psychiater erst nach 30 Therapiestunden ins Spiel. Im Delegationsmodell hingegen ist die fachärztliche Unterstützung und Zusammenarbeit ab Therapiebeginn sichergestellt.

Ausreichende klinisch-praktische Erfahrung in SIWFzertifizierten psychiatrisch-psychotherapeutischen Institutionen ist auch deshalb zwingend, weil im neuen Modell Anordnungen auch von Grundversorgern gemacht werden, die in der Regel wenig Erfahrung mit der Diagnostik und Therapie von psychischen Erkrankungen haben. Deshalb ist es eminent 
wichtig, dass psychologische Psychotherapeutinnen und Psychotherapeuten lernen, schwerere psychische Störungen sicher zu erkennen.

Nur so können die Indikationsqualität und die Anforderungen an Behandlungsqualität und -sicherheit von Beginn weg erfüllt und Fehlbehandlungen vermieden werden. Die klinische Erfahrung ist nicht zuletzt auch bei Kriseninterventionen und Notfallbehandlungen von entscheidender Bedeutung - also dort, wo heute tatsächlich Versorgungsengpässe bestehen, die der Bundesrat mit dem Anordnungsmodell beheben will.

\section{Unterfinanzierung}

Bei ungenügender klinischer Erfahrung werden sich psychologische Psychotherapeutinnen und -therapeuten zwangsläufig auf leichte Fälle konzentrieren. Es besteht die Gefahr, dass die ambulante psychiatrische Versorgung - niedergelassen und institutionell - mit dieser Umverteilung mehr schwere Fälle übernehmen muss. Das aktuelle Tarifsystem vergütet die ambulante Behandlung für schwer und komplex psychisch kranke Menschen allerdings nicht kostendeckend. Auch mit den heute schon vorhandenen kantonalen Subventionen in Form von gemeinwirtschaftlichen Leistungen können die institutionellen Ambulatorien nicht wirtschaftlich betrieben werden. Der finanzielle Druck auf die ambulante psychiatrische Behandlung schwer kranker Menschen wird steigen.

\section{Fachkräftemangel}

Wenn die ambulant tätigen Fachpersonen in den Institutionen sowie die niedergelassenen Psychiaterinnen und Psychiater zunehmend mit einer Konzentration von schwer psychisch erkrankten Menschen konfrontiert sein werden, sinkt die Attraktivität für die verantwortlichen Berufsgruppen - insbesondere für den ärztlichen und den pflegerischen Nachwuchs. Diese asymmetrische Lastenverteilung wird den Fachkräftemangel in den psychiatrischen Institutionen weiter verschärfen. Mit der heute vorgesehenen flächendeckenden, unregulierten Mengenausweitung ist die Gefahr einer Ressourcenverschiebung von schweren zu leichteren Fällen besonders gross.

\section{Kostenentwicklung}

Die vom Bundesamt für Gesundheit genannten Mehrkosten von 167 Millionen Franken sind deutlich zu tief geschätzt und basieren auf nicht repräsentativen Zahlen und Fakten. Gefordert ist eine verlässliche Zahlenbasis. Es reicht auch nicht aus, die Kostenentwicklung zu beobachten. Ohne wirksame Zulassungssteuerung durch die Kantone wird es in diesem Bereich zu einem unkontrollierten Wachstum der Gesundheitskosten ohne den angestrebten Gegenwert kommen - dies in Zeiten, in denen im Gesundheitswesen der Spardruck hoch ist.

\section{Prozess verlangsamen}

Ein halbes Jahr vor dem Systemwechsel vom Delegations- zum Anordnungsmodell bestehen noch immer fundamentale Unklarheiten in Bezug auf die gezielte Versorgung, die Sicherheit, die Qualität und die Kosten. Die vom Bundesrat vorgegebenen Ziele, die Engpässe in der psychiatrisch-psychotherapeutischen Versorgung zu beheben, bleiben unter diesen Voraussetzungen ein Wunschdenken. Wie sich nun zeigt, wäre angesichts der Komplexität und Tragweite des Systemwechsels eine KVG-Revision angezeigt gewesen.

Es besteht aber immer noch die Chance, den Systemwechsel auf dem Verordnungsweg so umzusetzen, dass die Engpässe in der Kinder- und Jugendpsychiatrie, in Randregionen, in der Krisen- und Notfallpsychiatrie sowie generell bei psychisch schwer erkrankten Menschen reduziert werden. Daher sollte der auf den 1. Juli 2022 geplante Systemwechsel auf einen späteren Zeitpunkt verschoben werden. So liessen sich die notwendigen Voraussetzungen schaffen, um eine Fehlversorgung ebenso wie ungerechtfertigte Mehrkosten $\mathrm{zu}$ verhindern. Fragen rund um die Zulassungssteuerung und die Weiterbildung der Psychologinnen und Psychologen sind zwingend zu klären, bevor das Anordnungsmodell in Kraft tritt. Die Gefahr ist sonst gross, dass Menschen mit schwereren psychischen Erkrankungen nicht adäquat behandelt werden, wenn die psychologischen Psychotherapeuten in der Weiterbildung nicht auf die neuen Bedingungen im Anordnungsmodell vorbereitet werden. Massgebend für die Umsetzung müssen die tatsächlich bestehenden Versorgungsprobleme sein, und es müssen die Qualitätsund Sicherheitsaspekte inklusive Finanzierung und Konzeption einer adäquaten klinischen Weiterbildung berücksichtigt werden.

\section{Das Wichtigste in Kürze}

- Per 1. Juli 2022 soll gemäss Bundesratsentscheid das Delegationsmodell für psychotherapeutische Behandlungen durch psychologische Psychotherapeutinnen und -therapeuten durch das Anordnungsmodell ersetzt werden. So sollen Versorgungsengpässe verhindert werden.

- Diese Änderung wirft aus Sicht der SGPP und SMHC Fragen in den Bereichen der Zulassung, klinischen Weiterbildung und Finanzierung sowie beim Fachkräftemangel und bei der Kostenentwicklung auf.

- $\quad$ Die SGPP und SMHC plädieren für eine zeitliche Verschiebung des Systemwechsels, damit diese Fragen angemessen geklärt und unnötige Mehrkosten verhindert werden. 